\title{
An Automated Framework of Inner Segment/Outer Segment Defect Detection for Retinal SD-OCT Images
}

\author{
Weifang Zhu ${ }^{1}$, Fei Shi ${ }^{1}$, Dehui Xiang ${ }^{1}$, Enting Gao ${ }^{1}$, Liyun Wang ${ }^{1}$, Haoyun $\mathrm{Chen}^{2}$, \\ Xinjian Chen $^{1 *}$ \\ 1School of Electrical and Information Engineering, Soochow University, Suzhou, China \\ ${ }^{2}$ Joint Shantou International Eye Center, Shantou University and the Chinese University of \\ Hong Kong, Shantou, China
}

\begin{abstract}
The integrity of inner segment/outer segment (IS/OS) has high correlation with lower visual acuity in patients suffering from blunt trauma. An automated 3D IS/OS defect detection method based on the SD-OCT images was proposed. First, 11 surfaces were automatically segmented using the multiscale 3D graph-search approach. Second, the sub-volumes between surface 7 and 8 containing IS/OS region around the fovea (diameter of $1 \mathrm{~mm}$ ) were extracted and flattened based on the segmented retinal pigment epithelium layer Third, 5 kinds of texture based features were extracted for each voxel. A KNN classifier was trained and each voxel was classified as disrupted or nondisrupted and the responding defect volume was calculated. The proposed method was trained and tested on 9 eyes from 9 trauma subjects using the leave-one-out cross validation method. The preliminary results demonstrated the feasibility and efficiency of the proposed method.
\end{abstract}

Keywords: IS/OS Defect, Segmentation, Graph-Search, KNN classifier.

\section{Introduction}

The retina is the sensory tissue in the back part of the eye, which is very important to human visual system. The retina has a basic structure consisting of three layers of nerve cells separated by two layers filled with synaptic connections [1]. The photoreceptors lie in the outermost cell layer and include two kinds of cells, rods and cones. The inner segment/outer segment (IS/OS) is the junction of inner segment and outer segment, which divides the photoreceptors into two parts. Recently, many studies have shown that the extent of the defect of the IS/OS junction is an important clinical indicator for the injury degree of photoreceptors, which may be closely associated with visual acuity in different eye diseases [2-9], although controversy remains [10-13].

Spectral-domain optical coherence tomography (SD-OCT) can produce high speed, high resolution, cross sectional 3D images, which is a powerful technology for the noninvasive assessment of retinal physiology and pathology [14]. With the increasing

${ }^{*}$ Corresponding author. Address: No. 1, Shizi Street, Suzhou, Jiangsu, P.R. China 215006. Tel.: +86-18260180695. Email: xjchen@ suda.edu.cn 
number of acquired SD-OCT volumes and their resolution, automatic assessment methods become increasingly desirable.

There are several researches focused on the quantitative measurements of the IS/OS junction defect area on the SD-OCT images [5, 6]. In [5], a semi-automated and linear area measurement for the IS/OS junction defect was proposed, which may involve subjective factors when selecting the defect margins. In [6], consideration of the irregular shape of IS/OS defect, an automated IS/OS junction defect margin detection and a weighted IS/OS defect area was calculated by the assumption that the shape of the defect was circular, which was unreasonable.

In this paper, we proposed an automated method for the IS/OS defect detection and the corresponding quantitative defect volume calculation, since the IS/OS junction is a membrane with small thickness in the photoreceptor. It may be of great level interest because of its potential to diagnose the diseases, evaluate the clinic treatment and prognosis and possibly predict visual outcomes in the patients with ocular trauma.

\section{Materials}

Nine eyes (6 right eyes and 3 left eyes) from nine subjects diagnosed with retinal trauma were included and underwent macular-centered SD-OCT imaging (Topcon, $512 \times 64 \times 480 \quad$ voxels, $11.72 \times 93.75 \times 3.50 \mu \mathrm{m}^{3}$, or $512 \times 128 \times 480$ voxels, $\left.11.72 \times 46.88 \times 3.50 \mu \mathrm{m}^{3}\right)$. The collection and analysis of image data were approved by the Institutional Review Board of Joint Shantou International Eye Center (JSIEC), Shantou University and the Chinese University of Hong Kong and adhered to the tenets of the Declaration of Helsinki. Because of its retrospective nature, informed consent was not required from subjects. The medical records and OCT database of JSIEC from February 2009 to December 2011 were searched and reviewed.

The IS/OS defect regions in 3-D SD-OCT were manually segmented slice by slice, under the directions of a highly experienced ophthalmologist. The marked results are considered as the ground truth.

\section{Methods}

The basic diagram for the IS/OS defect detection is shown in Fig. 1, which includes 3 steps: image preprocessing, volumetric feature extraction, KNN classifier training and testing. The details are as following.

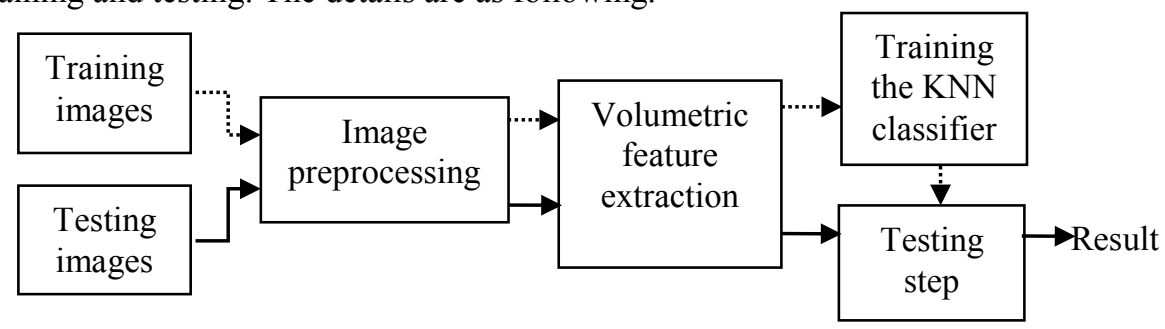

Fig. 1. Diagram of the automated IS/OS defect detection. 


\subsection{Imaging Preprocessing}

There are two steps in the image preprocessing: intra-retinal layer segmentation, sub-volume containing IS/OS region extraction and flatten.

\subsubsection{Intra-retinal Layer Segmentation}

Intra-retinal layer segmentation is important to analyze the retinal disorders such as the severity of eye trauma and macular edema formation. The SD-OCT volume was automatically segmented into 10 intra-retinal layers identified in Fig.2 using the multi-scale 3-D graph-search approach [15-18], producing 11 surfaces. The basic idea of this method is to detect the retinal surface in high-resolution sub-volume, which contains the surface and has been segmented at a lower resolution.

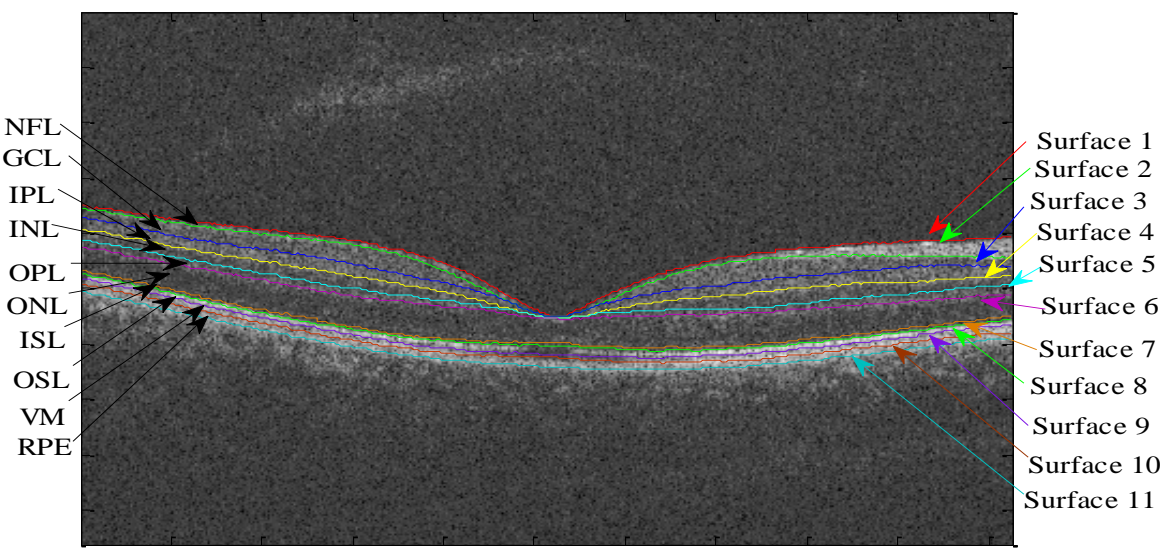

Fig. 2. Segmentation results of 11 surfaces (10 layers) in $X-Z$ image of the OCT volume, 10 layers are: nerve fiber layer (NFL), ganglion cell layer (GCL), inner plexiform layer (IPL), inner nuclear layer (INL), outer plexiform layer (OPL), outer nuclear layer (ONL), inner segment layer (ISL), outer segment layer (OSL), Verhoeff's membrane (VE), and retinal pigment epithelium (RPE)[19].

\subsubsection{IS/OS region extraction and flatten}

Once all the surfaces were detected, the sub-volume containing the IS/OS region between surface 7 and surface 8 was extracted and flattened based on the retinal pigment epithelium (RPE), i.e., surface 11, because of its robustness. If the IS/OS is disrupted around the central fovea, it will importantly implicate the patient's central visual acuity. In this primarily research, the sub-volumes between surface 7 and surface 8 and within a cylinder, which is centered at the fovea and with the diameter of $1 \mathrm{~mm}$ were our volumes of interest (VOIs), as shown in Fig. 3. 


\subsection{Feature Extraction}

Our method is a texture-based method to discriminate IS/OS defect by extracting texture features from VOIs. As shown in Table 1, 5 kinds of low-level texture features including normalized intensity, gradient, block mean, block standard deviation and block image entropy were calculated.

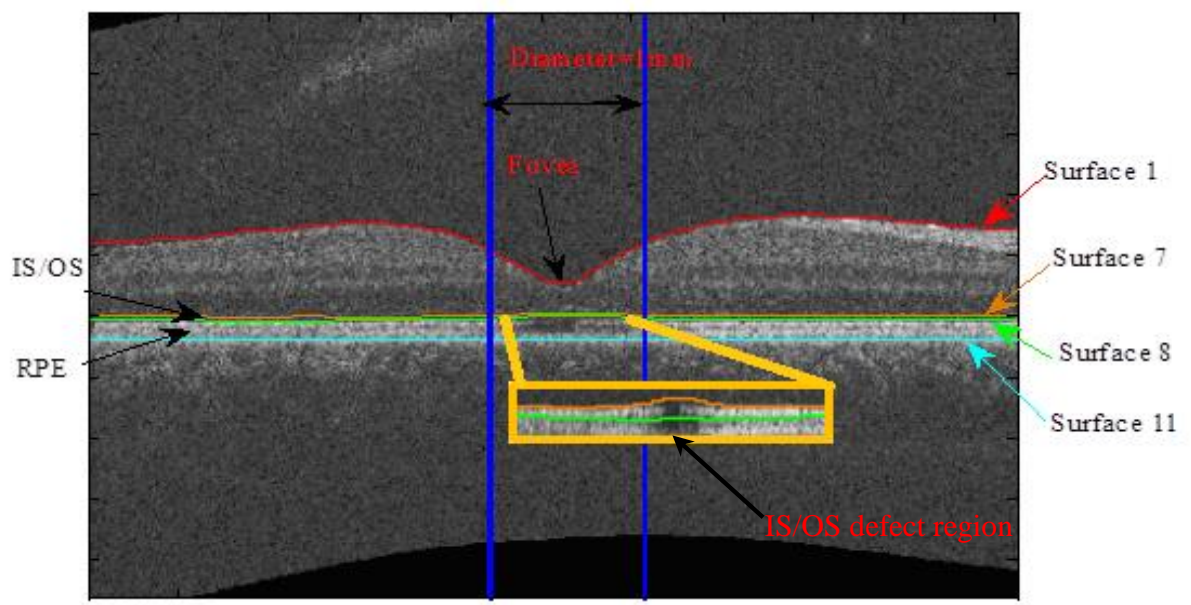

Fig. 3. The flattened OCT volume in X-Z image and the VOIs.

Table 1. Texture features extracted.

\begin{tabular}{|c|c|}
\hline Feature & Formula \\
\hline Normalized & $I_{\text {normalized }}(i, j, k)=\frac{I_{\text {original }}(i, j, k)-I_{\min }}{I_{\max }-I_{\min }} \times 255$ \\
\hline$I_{\text {normalized }}(i, j, k)$ & $\begin{array}{l}I_{\text {original }}(i, j, k) \text { represents the original intensity of voxel }(i, j, k) \text {, } \\
I_{\min } \text { and } I_{\max } \text { represents the minimum intensity value and the } \\
\text { maximum intensity value of the voxels in the VOIs, respectively. }\end{array}$ \\
\hline $\begin{array}{l}\text { Relative gradient } \\
G_{x}(i, j, k)\end{array}$ & $G_{x}(i, j, k)=\frac{\left|I_{\text {normalized }}(i+1, j, k)-I_{\text {normalized }}(i-1, j, k)\right|}{I_{\text {normalized }}(i, j, k)}$ \\
\hline $\begin{array}{l}\text { Relative gradient } \\
\qquad G_{y}(i, j, k)\end{array}$ & $G_{y}(i, j, k)=\frac{\left|I_{\text {normalized }}(i, j+1, k)-I_{\text {normalized }}(i, j-1, k)\right|}{I_{\text {normalized }}(i, j, k)}$ \\
\hline $\begin{array}{l}\text { Relative gradient } \\
G_{z}(i, j, k)\end{array}$ & $G_{z}(i, j, k)=\frac{\left|I_{\text {normalized }}(i, j, k+1)-I_{\text {normalized }}(i, j, k-1)\right|}{I_{\text {normalized }}(i, j, k)}$ \\
\hline $\begin{array}{l}\text { Block mean } \\
M_{\text {block }}(i, j, k)\end{array}$ & $M_{\text {block }}(i, j, k)=\frac{1}{27} \sum_{l=i-1}^{i+1} \sum_{m=j-1}^{j+1} \sum_{n=k-1}^{k+1} I_{n o r m a l i z e d}(l, m, n)$ \\
\hline \multirow[t]{2}{*}{$\begin{array}{l}\text { Block standard } \\
\text { deviation } \\
\operatorname{STD}_{\text {block }}(i, j, k)\end{array}$} & $S T D_{\text {block }}(i, j, k)=\sqrt{\sum_{l=i-1}^{i+1} \sum_{m=j-1}^{j+1} \sum_{n=k-1}^{k+1}\left(I_{\text {normalized }}(l, m, n)-M_{\text {block }}(i, j, k)\right)^{2}}$ \\
\hline & block $(2, j)$ \\
\hline
\end{tabular}




\begin{tabular}{c}
\hline$E N T_{\text {block }}=-\sum_{m=0}^{M-1} p(m) \log _{2} p(m)$ \\
Block image \\
entropy $E N T_{\text {block }}$ \\
$\mathrm{M}=256$ represents the total gray levels in the histogram, \\
histogram.
\end{tabular}

\subsection{Classifier Training and Testing}

With the features extracted from the 9 eyes from 9 subjects with blunt trauma, a KNN classifier was trained. The basic rule of the KNN classifier is finding the best category for the unknown sample according the known categories of its $\mathrm{K}$ neighbors. The optimal $\mathrm{K}$ was 10 . Each voxel in the testing VOIs was classified by using the trained KNN classifier point-by-point.

Because of the small amount of the experimental subjects, the feasibility and efficiency of the proposed method was tested by the leave-one-out cross validation.

\section{$4 \quad$ Results and Discussion}

The proposed method was tested on 9 eyes (6 right eyes and 3 left eyes) from 9 trauma subjects with IS/OS defect around the fovea. The true positive rate (TPR), the true negative rate (TNR) and the detection accuracy rate for each record were calculated using Formula (1), (2) and (3), respectively,

$$
\begin{gathered}
T P R=\frac{T P}{T P+F N} \\
T N R=\frac{T N}{T N+F P} \\
A C C=\frac{T P+T N}{T P+F P+T N+F N}
\end{gathered}
$$

In which, $T P, T N, F P$ and $F N$ represents true positive, true negative, false positive and false negative, respectively.

Fig. 4 showed the ground truth and detection results for Case 5. In Fig. 4, the figures in the top row were the five continuous slices, in which the manually marked ground truth was shown up as white. The figures in the bottom row showed the IS/OS detection results of the proposed method, in which white color represented the disrupted voxel. As we can see from Fig. 4, there were both some false positive errors and false negative errors. 


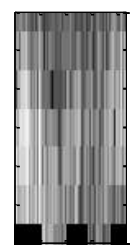

(a)

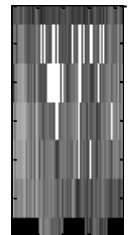

(f)

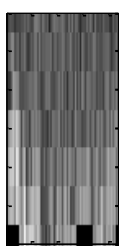

(b)

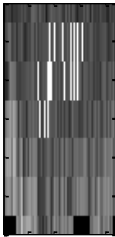

(g)

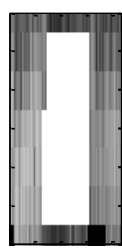

(c)

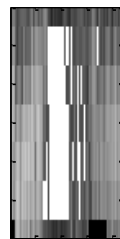

(h)

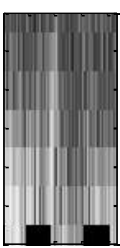

(d)

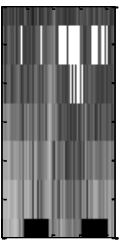

(i)

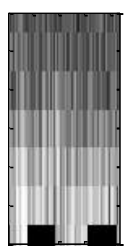

(e)

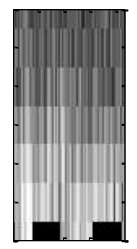

(j)

Fig. 4. The results of the IS/OS defect region detection. White voxel represented the disrupted voxel. The other grayscale represented the intensity value of the voxel in the VOIs. The upper were the ground truth and the lower were the detection results of the proposed method.

Table 2 showed the leave-one-out cross validation results. Fig. 5 graphically showed the corresponding results. As shown in Fig.5, some of the detection accuracy rates were low. The possible reasons may be: (1) the extracted features can not typically represent the essential attributes of the disrupted region and the nondisrupted region; (2) the KNN classifier can not work well; (3) in each VOIs, the numbers of the disrupted voxels are small and those of the non-disrupted voxels are large. The dramatic ratio between two kinds of voxels may be the negative factor for both the feature extraction and the classification.

Table 2. The results of the leave-one-out cross validation using KNN-classifier, in which "D" represents "disrupted" and "ND" represents "Non-disrupted".

\begin{tabular}{|c|c|c|c|c|c|c|c|}
\hline \multirow[b]{2}{*}{ Test data } & \multicolumn{2}{|c|}{ Ground truth } & \multicolumn{2}{|c|}{ Detection result } & \multirow{2}{*}{$\begin{array}{l}\text { TPR } \\
(\%)\end{array}$} & \multirow[b]{2}{*}{$\begin{array}{c}\text { TNR } \\
(\%)\end{array}$} & \multirow{2}{*}{$\begin{array}{c}\mathrm{ACC} \\
(\%)\end{array}$} \\
\hline & $\begin{array}{c}D \\
\left(\mu m^{3}\right)\end{array}$ & $\begin{array}{c}\text { ND } \\
\left(\mu \mathrm{m}^{3}\right)\end{array}$ & $\begin{array}{c}D \\
\left(\mu m^{3}\right)\end{array}$ & $\begin{array}{c}\text { ND } \\
\left(\mu \mathrm{m}^{3}\right)\end{array}$ & & & \\
\hline 1 & $6.056 \mathrm{E} 5$ & $1.722 \mathrm{E} 7$ & $4.768 \mathrm{E} 5$ & $1.444 \mathrm{E} 7$ & 0.629 & 0.839 & 0.830 \\
\hline 2 & 3.807E5 & $1.642 \mathrm{E} 7$ & $2.076 \mathrm{E} 5$ & $1.342 \mathrm{E} 7$ & 0.546 & 0.817 & 0.811 \\
\hline 3 & 6.027E6 & $7.698 \mathrm{E} 6$ & 5.001E6 & 2.013E6 & 0.830 & 0.273 & 0.518 \\
\hline 4 & 7.037E5 & $1.559 \mathrm{E} 7$ & $3.076 \mathrm{E} 5$ & $1.075 \mathrm{E} 7$ & 0.437 & 0.690 & 0.679 \\
\hline 5 & 6.614E5 & $1.722 \mathrm{E} 7$ & $5.306 \mathrm{E} 5$ & $1.370 \mathrm{E} 7$ & 0.802 & 0.796 & 0.796 \\
\hline 6 & $3.076 \mathrm{E} 5$ & $1.725 \mathrm{E} 7$ & $1.000 \mathrm{E} 5$ & $1.370 \mathrm{E} 7$ & 0.325 & 0.794 & 0.786 \\
\hline 7 & $7.575 \mathrm{E} 5$ & $1.623 \mathrm{E} 7$ & $5.037 \mathrm{E} 5$ & $9.655 \mathrm{E} 6$ & 0.665 & 0.595 & 0.598 \\
\hline 8 & $2.499 \mathrm{E} 5$ & $1.413 \mathrm{E} 7$ & $1.349 \mathrm{E} 5$ & $1.059 \mathrm{E} 7$ & 0.539 & 0.749 & 0.746 \\
\hline 9 & $6.975 \mathrm{E} 6$ & $7.391 \mathrm{E} 6$ & $4.534 \mathrm{E} 6$ & $4.245 \mathrm{E} 6$ & 0.650 & 0.574 & 0.611 \\
\hline
\end{tabular}




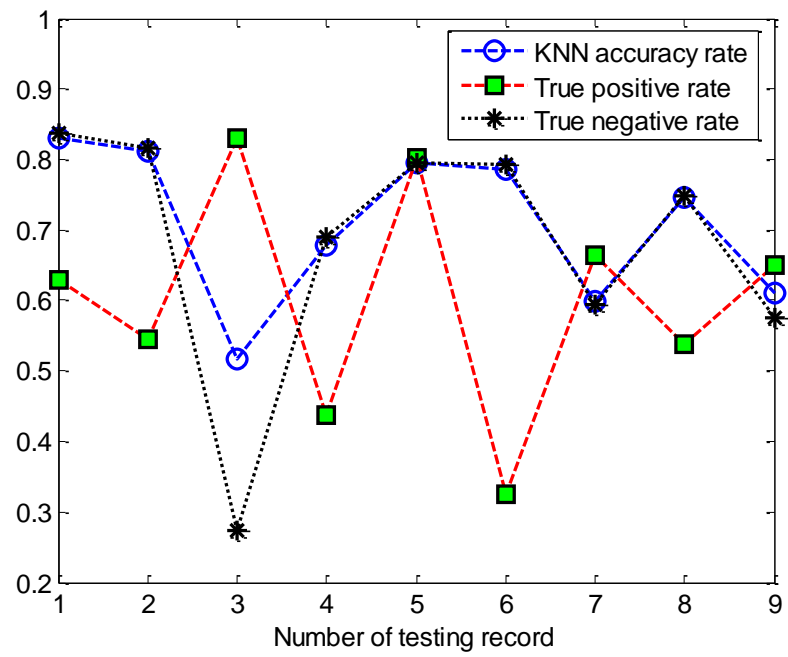

Fig. 5. True positive rate, true negative rate and the accuracy rate using $\mathrm{KNN}$ classifier for each testing record.

\section{Conclusions}

In this preliminary study, we have developed and evaluated an automated method to detect the 3D integrity of the IS/OS in patients with blunt trauma, which is a common disease in ophthalmic clinic and is primarily associated with visual acuity. The preliminary results showed that the defected region of IS/OS can be effectively detected by the proposed method. These results can give the ophthalmologists some constructive suggestions.

We have started the further study and are trying to detect the whole IS/OS region between surface 7 and surface 8 with the improved method, in which vessel exclusion should be considered. More texture based features will be extracted and different kinds of classifiers will be used for the promotion of the detection accuracy rate. Also more subjects are needed for testing and validating.

\section{References}

[1] K. Helga. "How the retina works: Much of the construction of an image takes place in the retina itself through the use of specialized neural circuits", American Scientist, 2003, January-February, 91: 28-35.

[2] M. Hangai, Y. Ojima, N. Gotoh, et al. "Three-dimension imaging of macular holes with high-speed optical coherence tomography", Opthalmology, 2007; 114(4): 763-773.

[3] M. Inoue, Y. Watanable, A. Arakawa, S. Sato, S. Kobayashi and K. Kadonosono. "Spectraldomain optical coherence tomagraphy images of inner/outer segment junctions and macular 
hole surgery outcomes", Graefes Archive Clinical and Experimental Opthalmology, 2009; 247:325-330

[4] O. Jaeryung, E. S. William, W.F.J. Harry, G. Giovanni, and L. Brandon. "Photoreceptor inner/outer segment defect imaging by spectral domain OCT and visual prognosis after macular hole surgery", Investigative Ophthalmology \& Visual Science, 2010; 51(3): 16511658.

[5] K. Sayanagi, Y. Ikuno, K. Soga, and Y. Tano. "Photoreceptor inner and outer segment defects in Myopic Foveoschisis", American Journal of Ophthalmology, 2008; 145(5): 902908.

[6] R.F. Spaide, H. Koizumi, and K.B. Freund. "Photoreceptor outer segment abnormalities as a cause of blind spot enlargement in acute zonal occult outer retinopathy-complex diseases", American Journal of Ophthalmology, 2008; 146(1): 111-120.

[7] T. Baba, S. Yamamoto, M. Arai, et al. "Correlation of visual recovery and presence of photoreceptor inner/outer segment junction in optical coherence images after successful macular hole repair", Retina, 2008; 28(3): 453-458.

[8] N. Kitaya, T. Hikichi, H. Kagokawa, A. Takamiya, and A. Yoshida. "Irregularity of photoreceptor layer after successful maculor hole surgery prevents visual acuity improvement", American Journal of Ophthalmology, 2004; 138(2): 308-310.

[9] N.L. Gomes, V.C. Greenstein, J.N. Carlson, et al. "A comparison of fundus autofluorescence and retinal structure in patients with stargardt disease", Investigative Ophthalmology \& Visual Science, 2009; 50(8): 3953-3959.

[10] N. Villate, J.E. Lee, A. Venkatraman, W.E. Smiddy. "Photoreceptor layer features in eyes with closed macular holes: optical coherence tomography findings and correlation with visual outcomes", American Journal of Ophthalmology, 2005; 139(2): 280-289.

[11] M. H. Suh, J.M. Seo, K.H. Park, H.G. Yu. "Associations between macular findings by optical coherence tomography and visual outcomes after epiretinal membrane removal", American Journal of Ophthalmology, 2009; 147(3): 280-289.

[12] L.K. Chang, H. Koizumi, R.F. Spaid. "Disruption of the photoreceptor inner segment-out segment junction in eyes with macular holes", Retina, 2008; 28(7):969-975.

[13] A.A. Moshfeghi, Jr H.W. Flynn, S.G. Elner, et al. "Persistent outer retinal defect after successful macular hole repair", American Journal of Ophthalmology, 2005; 139(1): 183184.

[14] C. Cukras, Y.D. Wang, C.B. Meyerle, F. Forooghian, E.Y. Chew, and W.T. Wong, "Optical coherence tomography-based decision making in exudative age-related macular degeneration: Comparison of time- vs spectral-domain devices”, Eye, 2009.

[15] K. Li, X. Wu, D.Z. Chen, M. Sonka, "Optimal surface segmentation in volumetric imagesa graph-theoretic approach", IEEE Transactions on Pattern Analysis and Machine Intelligence, 2006, 28:119-134.

[16] M.K. Garvin, M.D. Abràmoff, X. Wu, S.R. Russell, T.L. Burns, M. Sonka, "Automated 3D intrarential layer segmentation of macular spectral-domain optical coherence tomography images", IEEE Transactions on Medical Imaging, 2009, 28:1436-1447.

[17] M.K. Garvin, M.D. Abràmoff, R. Kardon, S.R. Russell, X. Wu, M. Sonka, "Intraretinal layer segmentation of macular optical coherence tomography images using optimal 3-D graph search", IEEE Transactions on Medical Imaging, 2008, 27:1495-1505.

[18] K. Lee, M. Niemeijer, M. K. Garvin, Y.H. Kwon, M. Sonka, M.D. Abràmoff, "Segmentation of the optic disc in 3-D OCT scans of the optic nerve head", IEEE Transactions on Medical Imaging, 2010, 29:159-168.

[19] C. Gerth, R. J. Zawadzki, J. S. Werner, and E. Hèon, "Retinal microstructure in patients with efemp1 retinal dystrophy evaluated by Fourier domain OCT", Eye, 2009, 23(1): 480483. 